\title{
Erratum to: "Overexpression, Homology Modeling and Coenzyme Docking Studies of the Cytochrome P450nor2 from Cylindrocarpon tonkinense" [Molecular Biology 50, 320 (2016)]
}

\author{
N. Lia, b, 2, Y. Z. Zhang ${ }^{a, 2}$, D. D. Li ${ }^{a}$, Y. H. Niu ${ }^{a}$, J. Liu ${ }^{a}$, S. X. Li ${ }^{a}$, \\ Y. Z. Yuan ${ }^{a}$, S. L. Chen ${ }^{a}$, H. Geng ${ }^{a}$, and D. L. Liu ${ }^{a, *}$ \\ ${ }^{a}$ Hubei Key Laboratory of Genetic Regulation and Integrative Biology, School of Life Science, \\ Central China Normal University, Wuhan, 430079 China \\ ${ }^{b}$ Key Laboratory of Crops with High Quality and Efficient Cultivation and Security Control, Yunnan Higher Education \\ Institutions, College of Life Science and Technology, Honghe University, Mengzi, 661100 China \\ *e-mail:deliliu2013@163.com; ldl@mail.ccnu.edu.cn \\ (Submitted July 15, 2016; accepted for publication July 15, 2016)
}

DOI: $10.1134 / \mathrm{S} 0026893316050198$

The list of authors should read as follows:

N. Li ${ }^{a, b, 2}$, Y. Z. Zhang ${ }^{a, 2}$, D. D. Li ${ }^{a}$, Y. H. Niu ${ }^{a}$, J. Liu ${ }^{a}$, S. X. Li ${ }^{a}$, Y. Z. Yuan ${ }^{a}$, S. L. Chen ${ }^{a}$, H. Geng ${ }^{a}$, and D. L. $\mathrm{Liu}^{a, *}$

${ }^{2}$ These authors contributed equally. 